\title{
The Energy Consumption Differences between Walking and Running at the Same Speed
}

\author{
Meijuan Wang ${ }^{1, a}$, Feng Liu ${ }^{1, b}$ \\ ${ }^{1}$ School of Physical Education, Shaanxi Normal University, Shaanxi 710119, China; \\ a1536946220@qq.com, biufeng668@snnu.edu.cn
}

Keywords: walking; running; energy consumption

\begin{abstract}
In this study, we used the way of reserve heart rate to explore the energy consumption of the fitness crowd when walking and running under the same condition of speed, and to provide the reference to the fitness crowd to choose the exercise intensity and sports mode rationally. Using six male PE majors in Shaanxi Normal University as experimental subjects, the study used the one-dimensional force-measuring treadmill of H/P/COSMOS Gaitway to measure the speed and the POLAR S610i to measure the heart rate. In this study, speed were $4.8 \mathrm{~km} / \mathrm{h}$ and $6.4 \mathrm{~km} / \mathrm{h}$, test time was 20 minutes. The study finds that when the speed is $4.8 \mathrm{~km} / \mathrm{h}$, the energy consumption of running is higher than the energy consumption of walking; when the speed is $6.4 \mathrm{~km} / \mathrm{h}$, the situation is the same as the former. As a result, at the same speed, running will be more conducive to energy consumption, so as to achieve fitness effects.
\end{abstract}

\section{Introduction}

With the rapid development of society, the quality of people's life significantly improved. While enjoying their daily lives, a series of chronic diseases such as obesity, hypertension and so on, based on this public fitness awareness has been improved. The survey showed that the number of sports increased from 366 million to 435 million from 2009 to 2016. Walking and running account for 6.7\% of the total population of the country [1]. For most people, running was the most common way of activities. In recent years, researchers have studied energy consumption in walking and running from different perspectives, which were mainly reflected in the comparison of energy consumption at different speeds and the energy consumption of overweight people, while the comparative studies on the energy consumption of walking and running at the same speed were rare. Therefore, this study intended to provide a powerful theoretical basis for people to choose reasonable exercise methods by comparing the energy consumption of walking and running at the same speed.

\section{Methods}

All the tests were completed in Shaanxi Normal University of Sport, Sports Performance Research Centre (Shaanxi, People's Republic of China). The six testers in this study were undergraduate who majored in physical education at least 1 year. The study were collected in 6 participants (mean height: 176.5 \pm 3.73 , mean weight: $66.08 \pm 8.60$ ), the maximum heart rate controlled (189.8 \pm 0.61$)$, the resting heart rate controlled $(72.83 \pm 4.26)$, the basal heart rate controlled $(64.17 \pm 2.48)$. The study used the reserve heart rate method, the following steps are as follows: (1) estimate the maximum heart rate formula is 205.8-0.685*Age (2) calculated using Carbon formula\% HRR Work HR = (Maximum heart rate - Quiet heart rate) *\% HRR + Quiet Heart rate (3) the above two formulas calculate the percentage of reserve heart rate. Since the percentage of reserve heart rate can reflect the energy consumption, we used the reserve heart rate percentage to reflect the energy consumption in this experiment. This study was approved by the local ethical committee of Shaanxi Normal University, and all participants gave informed written consent prior to testing.

First of all, we kept all the six subjects informed of the items related to the experiment, then measured their resting heart rate, height and weight. And five minutes should be given to the testers to 
get familiar with the tread mill. The experiment will not be started until they come to a stable status. Requisite rest will be given to each tester after one test is done so as to ensure the reliability of the experiment. The way to identify a "stable status" were by monitoring the resting heart rate and asking how the subjects feel.

Treadmill speed setting: the speed of walking and running were $4.8 \mathrm{~km} / \mathrm{h}$ and $6.4 \mathrm{~km} / \mathrm{h}$ respectively. The walking and running adopt the sequence of first walking and second running, each speed level test time is 20 minutes, The steady-state time is set to 3 minutes, used the heart rate of $2 \sim 20$ minutes for statistical calculation. When walking and running in exchange, adequate rest should be stay for, and the experiment should be stopped immediately when the subjects were tired or had no physical strength.

Statistical analysis was carried out used SPSS 20.0 and Microsoft office excel. One-way analysis of SPSS 20.0 was used to calculate the mean value and the variance.

\section{Results}

\subsection{Average heart rate of the subjects}

During the experiment, the heart rate was recorded every two minutes. To ensure the validity of the data, we selected the heart rate from 2 to 20 and calculated the average heart rate, as shown in Table 1.

Table 1 Average heart rate of subjects during walking and running

\begin{tabular}{lcccc}
\hline Velocity $\mathrm{km} / \mathrm{h}$ & $4.8($ walk $) / \mathrm{bpm}$ & 4.8 (run)/bpm & $6.4($ walk $) / \mathrm{bpm}$ & 6.4 (run)/bpm \\
\hline 1.YUAN & 104 & 102 & 80 & 103 \\
2..LI & 102 & 105 & 98 & 120 \\
3..LIU & 80 & 98 & 110 & 117 \\
4..ZHANG & 103 & 120 & 117 & 137 \\
5..LIU & 104 & 116 & 112 & 134 \\
6..LANG & 107 & 120 & 117 & 139 \\
\hline
\end{tabular}

Table 1 shows that the changes in mean heart rate during walking and running in 6 subjects at 4.8 $\mathrm{km} / \mathrm{h}$ and $6.4 \mathrm{~km} / \mathrm{h}$, respectively. Table 1 shows that at a speed of $4.8 \mathrm{~km} / \mathrm{h}$, the average heart rate showed a walking lower than the running, and at a speed of $6.4 \mathrm{~km} / \mathrm{h}$ the situation is also the same. Therefore, we can infer that the running energy consumption is higher than the walking at the same speed.

\subsection{Subject reserve heart rate percentage}

Table 2 subjects reserve heart rate percentage situation

\begin{tabular}{lcccc}
\hline Velocity $\mathrm{km} / \mathrm{h}$ & $4.8($ walk $) / \mathrm{bpm}$ & 4.8 (run)/bpm & $6.4($ walk $) / \mathrm{bpm}$ & 6.4 (run) $/ \mathrm{bpm}$ \\
\hline 1.TUAN & 24.10 & 21.63 & 19.06 & 23.17 \\
2.LI & 21.63 & 30.16 & 26.53 & 32.58 \\
3.LIU & 19.06 & 26.53 & 32.19 & 36.33 \\
4.ZHANG & 23.17 & 32.58 & 36.33 & 53.80 \\
5.LIU & 22.26 & 31.27 & 35.39 & 52.73 \\
6.LANG & 24.14 & 38.24 & 39.27 & 57.35 \\
\hline
\end{tabular}

Table 2 shows that the running is higher than walking of energy consumption when the speed of $4.8 \mathrm{~km} / \mathrm{h}$, and the increase is more obvious. When the speed is $6.4 \mathrm{~km} / \mathrm{h}$, the situation is also the same. The speed of $6.4 \mathrm{~km} / \mathrm{h}$ and $4.8 \mathrm{~km} / \mathrm{h}$ go run compared to go higher than the energy run, this phenomenon shows that the faster the muscle activity is intense, the energy consumption is high. in addition, increased stride length and stride frequency helps to increase the utilization rate of fat and sugar, speed up the subjects relative increase stride frequency and length therefore, energy consumption increases, the speed of movement is determinants of human walking and running energy 
consumption[2-5].

\section{3 energy consumption contrast a speed of $6.4 \mathrm{~km} / \mathrm{h}$}

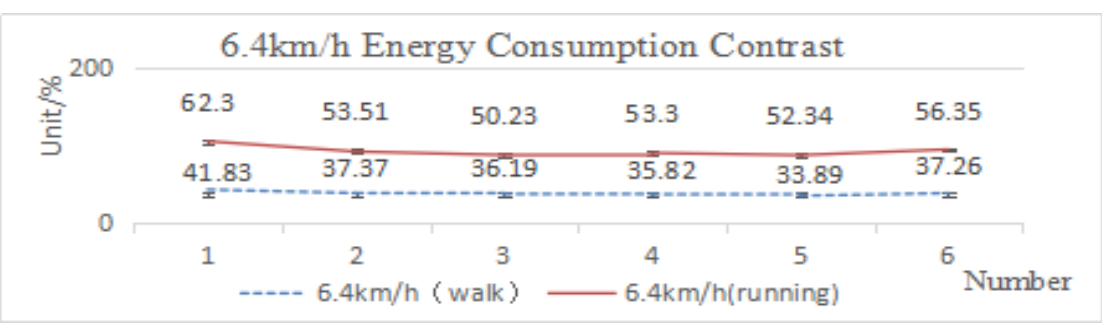

Fig. 1 energy consumption contrast a speed of $6.4 \mathrm{~km} / \mathrm{h}$

Fig. 1 shows that the running energy was greater than the walking, and there was a significant difference. This is because their movement mechanism is different when the body was walking, both feet will not leave the ground at the same time, at least one side of the lower limbs to support the body weight, and the existence of bilateral lower limbs supporting the body at the same time load-bearing stage; Side of the leg at the same time the load-bearing stage disappears, there have been two feet off the ground at the same time stage, the end of the two-legged into the stage when the unilateral leg support stage, the support leg to bear more than their own weight, which made the leg muscles do work increase.

\subsection{The number of training and the relationship between energy consumption}

Influencing factors of energy expenditure may be related to the number of training of their subjects, the training times of 6 subjects after investigation are as shown in Table 3.

Table 3 subjects training times

\begin{tabular}{ccccccc}
\hline Experimenter & YUAN & LI & LIU & ZHANG & LIU & LANG \\
\hline Times/week & 1 2times & 4times & 5 6times & 3times & 3 4times & 1 2times \\
\hline
\end{tabular}

In Table 3, the more times of training the energy consumption is relatively less, and because their lack of training so the energy consumption is relatively high. Subjects, Zhang is lighter, but its energy consumption is higher, the reason is that the training times are less, resulting in its poor ability to adapt to their body, a little speed in the experiment to accelerate its energy consumption will increase; and Liu although the weight was relatively big, due to frequent movements, leading to the muscle tissue to increase, the fat tissue decrease, so its weight is bigger, but less energy consumption. The results of this study show that those who regularly participate in sports training and are experienced in adapting to exercise load have a strong adaptability to the exercise load.

\subsection{BMI index and the relationship between energy consumption}

Studies have shown that the BMI index is an important factor to energy consumption. BMI index refer to as the body mass index and known as body mass index. Body Mass Index was divided by the weight of kilograms and the square of the number of height drawn figures, it is commonly used in the world to measure body fat level and a healthy standard. Because BMI is simple and practical to reflect generalized overweight and obesity, BMI is used to determine more accurate. Table 5 shows the BMI index of six subjects subject to follow-up.

Table 4 Subject BMI values

\begin{tabular}{ccccccc}
\hline Experimenter & YUAN & LIU & LI & LIU & LANG & ZHANG \\
\hline Weight $/ \mathrm{kg}$ & 75 & 70 & 70 & 66.5 & 65 & 50 \\
BMI kg/m2 & 24.77 & 20.90 & 22.34 & 20.99 & 20.99 & 16.70 \\
\hline
\end{tabular}

Note: BMI = body weight $(\mathrm{kg}) \div$ (height $(\mathrm{m}) \times$ height $(\mathrm{m})$ ); Less than 19.8 Skin less than $24.2 \sim 25.0$ Mild and obese;Less than $19.8 \sim$ 24.2 Ordinary 25.0 or more obese

In table 4, Yuan belongs to mild obesity and the energy consumption is relatively high, so body weight is an important factor leading to their consumed more energy. Zhang is thin and its energy 
consumption belongs to the middle number of subjects, but its training times are few, so the energy consumption not too low. Other subjects are all normal.

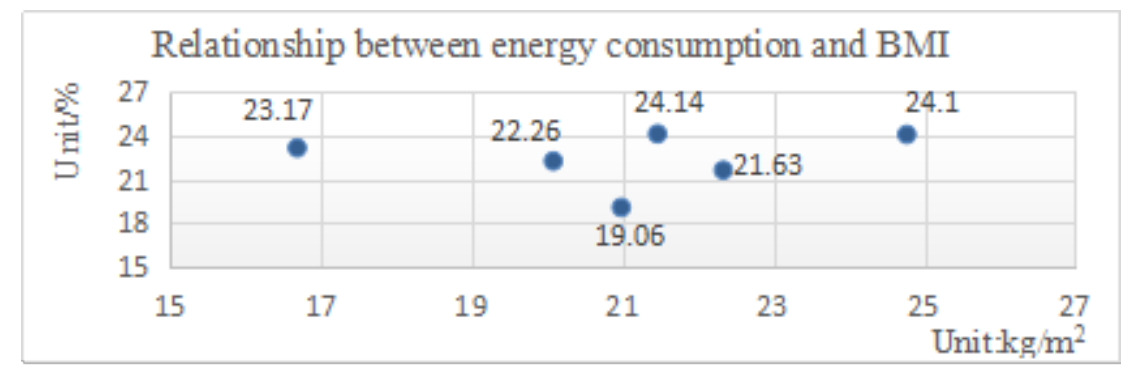

Fig. 2 relationship between BMI and energy consumption of $4.8 \mathrm{~km} / \mathrm{h}$

Fig. 2 shows that when the BMI is higher the energy will be more consumption, but not statistically significant. As a result, its energy consumption is relatively high. Yuan which is more obvious, due to its relatively large body weight led to relatively high BMI, resulting in its relatively high energy consumption, while Liu BMI is relatively low, so its energy consumption is also relatively Lower, the main factor is the BMI does not take into account the proportion of fat, so people with a larger BMI index due to less body fat resulting in lower energy consumption.

\section{Conclusion}

In the $4.8 \mathrm{~km} / \mathrm{h}$ and $6.4 \mathrm{~km} / \mathrm{h}$ two speed levels, running energy consumption is higher than walking energy consumption, so fitness weight loss exercise in order to increase the consumption of energy is recommended to choose the running gait mode of exercise.

BMI value is higher, the greater the weight, the more the energy consumption, but does not have statistical significance.

Training more energy consumption is relatively less, but usually lack of training its energy consumption is relatively large. Therefore, the number of subjects training affects how much their energy expenditure.

\section{References}

[1] Tao Zhuang, Bo Sun. Comparison of young men walking and running energy consumption. Chinese Journal of Tissue Engineering Research, 2012,37: 6937-6941.

[2] Bo Sun, Yu Liu, Haipeng Li. Study on the relationship between treadmill running and energy consumption and movement speed .Journal of Physical Education, 2012,09: 17-22.

[3] Sun Park, Yu Liu, Haipeng Li. A Comparative Study of Gait between Treadmill and Ground .Shanghai Sports Institute, 2012,06: 47-51.

[4] Chongmin Jiang, Shumin Qiu, Hua Wang. Comparative study on treadmill exercise and treadmill environmental energy consumption during walking and running. Journal of Physical Education, 2011,07: 30-36.

[5] Shufang Yue. Overweight adult women walking and running energy consumption comparison. Beijing Sport University, 2015. 